POLLACK PERIODICA

An International Journal for Engineering and Information Sciences

DOI: $10.1556 / 606.2017 .12 .1 .5$

Vol. 12, No. 1, pp. 59-67 (2017)

www.akademiai.com

\title{
PROCESS NETWORK SOLUTION OF A CLOTHING MANUFACTURER'S PROBLEM
}

\author{
Zsolt ERCSEY \\ Department of System and Software Technology \\ Faculty of Engineering and Information Technology, University of Pécs \\ Boszorkány út 2, 7624 Pécs, Hungary, e-mail: ercsey@mik.pte.hu
}

Received 11 December 2016; accepted 23 January 2017

\begin{abstract}
The current work focuses on a Hungarian clothing manufacturer's problem. First the industrial problem is presented; its corresponding critical pass method graph is depicted. To answer all emerging questions with respect to alternative possibilities, a large number of critical pass method problems have to be solved cumbersomely. Instead, first this graph is transformed into a process network. Alternatives specified by mainly financial necessities as well as human resource constraints can now be easily managed, namely where specific activities can be performed in different ways by various employee having different qualifications, requiring different durations and obviously respective costs can be considered within this model. These separate cases can commonly be handled within the resultant sole process network and the corresponding mathematical programming model.
\end{abstract}

Keywords: Project scheduling, Critical pass method, Process network, Alternatives

\section{Introduction}

A broadly used project management technique to algorithmically develop the scheduling of activities, the Critical Path Method (CPM) has its origins in the late 1950s. CPM is commonly used for construction projects, for product development or for software engineering purposes. First, when the set of activities with known duration times and known dependencies between the activities are given, an activity network is developed. Then, with logical end points, for example as deliverables or milestones CPM calculates the longest path of the planned activities together with their earliest starts and latest finish that each activity can start or finish without lengthening the 
overall project. In other words, the critical path is a series of depending activities adding up the longest overall duration of the project. Additionally, other paths within the network with shorter overall durations than the critical path are non-critical paths. There are activity-on-node and activity-on-arc representation of the CPM and there are transformation methods from one into the other illustrating that these representations are equivalent. For further details of the problem definition of the CPM please consider the work of Chanas and Zielinski [1].

Recent advances include the works Guerriero and Talarico [2] proposing a method to find the critical path in a deterministic activity-on-the-arc network with three different types of time constraints under consideration; while $\mathrm{Li}$ et al. [3] introduced a two-stage minimum risk problem and developed a hybrid algorithm by combining a dynamic programming method with binary particle swarm optimization. Szendröi [4] developed a robust hybrid algorithm for the multi-mode resource-constrained project scheduling problem, where a harmony search algorithm was combined with a 'head-tail' local search procedure based on a Mixed Integer Linear Programming (MILP) formulation. Csébfalvi and Láng [5] presented an improved hybrid method for the resource-constrained project scheduling problem with discounted cash flows; their MILP formulation is relaxed with linear approximation. Danka [6] presented an appropriate method from the statistical point of view for comparing different heuristics or meta-heuristics resource-constrained project scheduling problems. Liu et al. [7] developed an activity-list-based nested partitions algorithm for solving the resourceconstrained project scheduling problem. To improve the search efficiency, a partial double justification was also employed as local search mechanism. Please note that the structural properties and the consequences that can be drawn from structural investigations are used with extreme limitation in this field. For example, CPM techniques order the resources to the activities but this order does not appear in the graph representation. Moreover, some of the resource constrained project scheduling methods does not even consider any structural properties of the original problem.

Used basically in the chemical industry, Process Network Synthesis (PNS) is an optimization methodology, which utilizes graph theoretical approaches and combinatorial techniques. The first focus is on the structure generation of the problem under consideration, namely a bipartite directed graph representing the structure of the process system is generated. Besides this unique representation, an underlying system of axioms is also used to derive theorems to generate the potentially feasible structures as well as the so-called maximal structure which includes all feasible solution structures. After displaying all the structural properties of the problem, the consequences are exploited in the resulting mathematical programming model, in other words, this method handles the set of feasible solutions within one model and a single solution process. For further details, please consider the works of Friedler et al. [8] and [9].

Recent advances expanded the use of the process network synthesis methodology into other fields as well. In the field of business process modeling, for the analysis of workflow models the robust extension of the combinatorial PNS model was introduced by Tick et al. [10]; with an exponential time B\&B algorithm for the general case as well as a faster polynomial time algorithm for the special thin hierarchical problems. Indicating the importance of structural properties, for separation network synthesis problems where simple and sharps separators are considered with multiple feed-streams 
and multicomponent product-streams Kovács et al. [11] gave an algorithm to generate the exact super-structure, which always include the optimal separation network of the problem while it does not include any unnecessary operating units; and later Kovács et al. [12] introduced a method to generate the complete super-structure and the corresponding mathematical programming model necessary for the separation network synthesis problems with linear cost function. Due to the flexibility of the process network synthesis methodology Mandl et al. [13] examined energy networks, König et al. [14] dealt with designing flexible processes, e.g. flexible supply chains; Imreh and Kovács [15] investigated pollution control, Kollmann et al. [16] considered the integration of wastewater systems and energy networks.

\section{Suit jacket manufacturing problem}

A clothing manufacturer at the southern region of Hungary produces suit jackets, where the jackets differ in terms of their material patterns as well as in terms of the frequency of the quality control. One type of jacket manufacturing process is presented in this paper from the incoming raw material until the product shipping into the warehouse. There are 100 persons working in the process at a time depending on the workload. Since most of the production line is linear some activities were combined in groups for simplicity in this paper. The activity groups together with their durations are given in Table $I$. It is interesting to note that since there are multiple atomic activities within the activity groups densely following each other, i.e. they are strongly linked together, smaller quality problems are immediately corrected along the production process and therefore apart from material defects there is no production waste at the level of finished products.

Financial necessities as well as human resource constraints popped up the question of potential alternatives at the manufacturing process described above; namely when an activity or a set of activities may be replaced or performed by another activity with different duration, cost, resources necessary etc. As an illustration, three activities were considered for this manufacturing process, where alternatives were added, namely activities no. A3 'sleeve', no. A7 'cotton preparation', and no. A16 'button hole model' may be performed in two different ways. Please note that these activities and their corresponding alternative activities are Pareto optimal, namely where the duration is longer, the corresponding cost is higher, as it can clearly be seen in the data of Table I.

\section{Structure representation}

The solution method proposed in the present paper has the following steps:

i) generation of the CPM graph;

ii) mapping the CPM graph into a process network;

iii) adding the alternatives; and

iv) generation and solution of the mathematical programming model. 
Table I

Production steps/activities with their dependencies, durations, and costs; alternatives are indicated in brackets.

\begin{tabular}{|c|c|c|c|c|}
\hline \multicolumn{2}{|c|}{ Production steps / Activities } & \multirow{2}{*}{ Dependencies } & \multirow{2}{*}{ Duration (sec) } & \multirow{2}{*}{ Cost (HUF) } \\
\hline No. & Description & & & \\
\hline A1 & Sweating tiles & & 115 & 317 \\
\hline $\mathrm{A} 2$ & Collar & & 301 & 396 \\
\hline A3 & Sleeve & & $861(1000)$ & $706(362)$ \\
\hline A4 & Back & & 142 & 202 \\
\hline A5 & Pocket & & 892 & 533 \\
\hline A6 & Pocket cover & & 726 & 697 \\
\hline A7 & Cotton preparation & & $318.5(466)$ & $472(249)$ \\
\hline A8 & Chest & & 619 & 219 \\
\hline A9 & Cotton production & $\mathrm{A} 7, \mathrm{~A} 8$ & 2017.94 & 730 \\
\hline A10 & Side pocket & A6, A9 & 904 & 264 \\
\hline A11 & Front production & A5, A10 & 259 & 667 \\
\hline A12 & Side production & A4, A11 & 666 & 786 \\
\hline A13 & Sleeve production & A3, A12 & 454 & 219 \\
\hline A14 & Sleeve, shoulder, collar & A2, A13 & 628 & 589 \\
\hline A15 & Collar, sleeve, bottom, front & A1, A14 & 628 & 289 \\
\hline A16 & Button hole, model & A15 & $1101.56(246)$ & $195(370)$ \\
\hline
\end{tabular}

Generation of the CPM graph can be done based on the data given in Table I. As described in details in Vincze et al. [17] this CPM graph can be mapped into a process network. The advantages of this transformation are the following: alternatives can be easily added in the process network as additional structural information, resources belonging to the activities can be represented directly in the process network, time together with costs can be handled within one model and one solution, moreover not only the optimal solution network but all potentially feasible solution networks can be generated algorithmically and these can be ranked according to a predetermined objective function. As it can be clearly seen on the listed advantages, structural representation has its importance. Please note that Fig. Ia contains the activity on arc CPM graph of the suit jacket manufacturing problem, while Fig. $1 b$ contains the extended problem, where the alternative activities discussed above are already considered.

Please note that in the CPM graph in Fig. Ia there are some dummy arrows relating to the dependencies. Usually dummy arrows and dummy activities of the CPM graph do not need to be represented in the process network, since their time, costs etc. are considered to be zero. On the other hand, operating unit 'close' introduced for technical 
reasons simplifies the mathematical programming model; therefore it is included in Fig. $1 \mathrm{~b}$ even though the end product of the operating unit 'Button hole, model' equals to the finish of all activities.

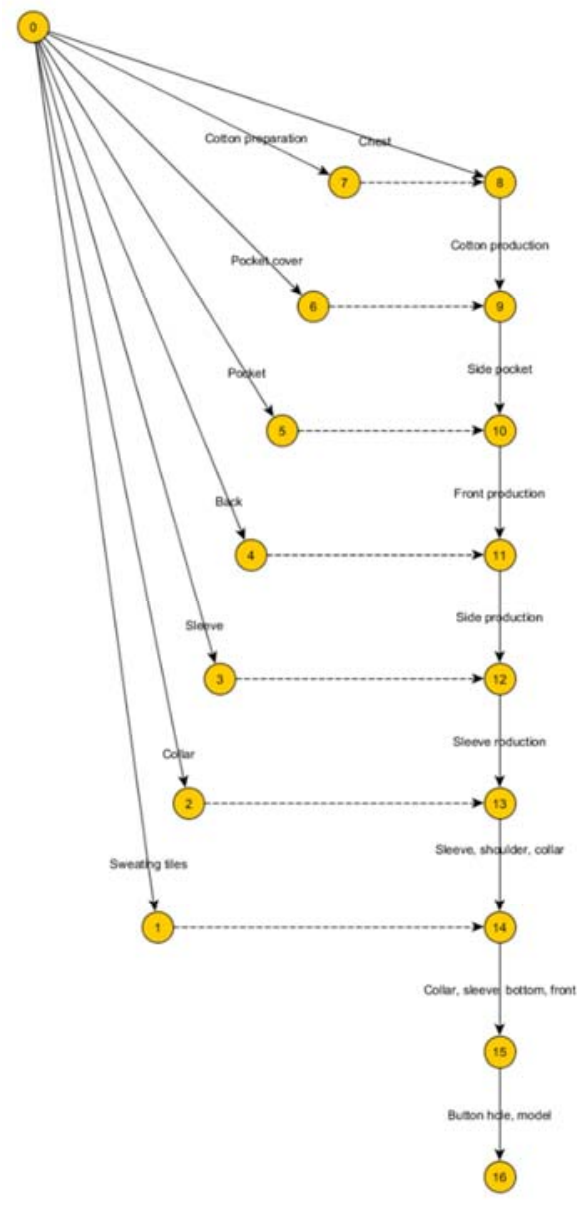

a) The activity on arc CPM graph

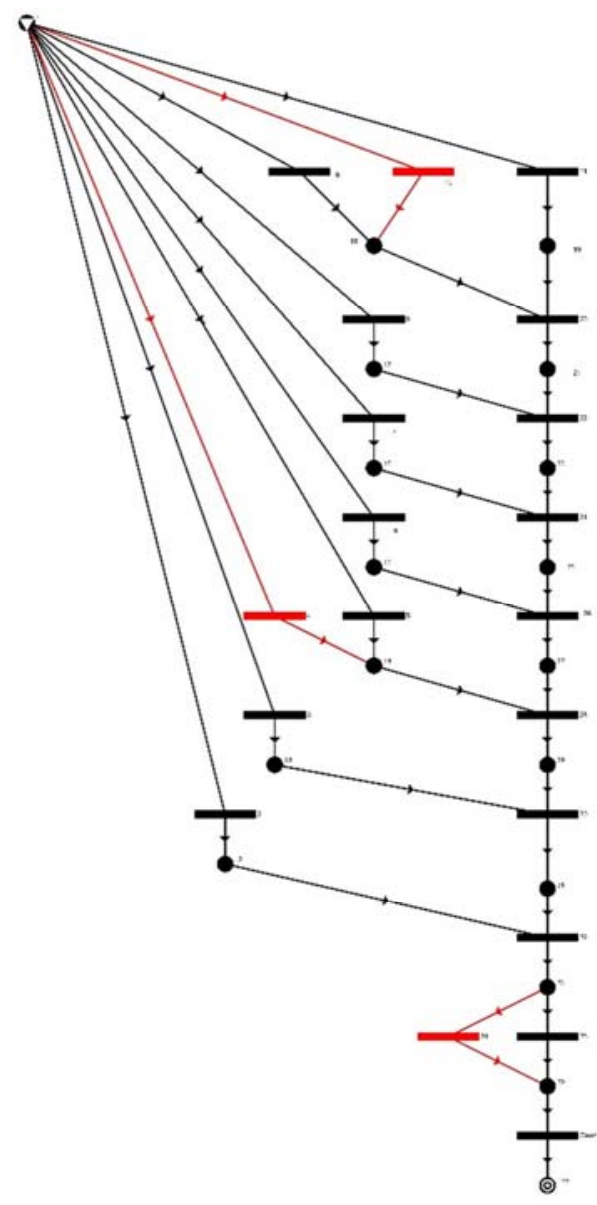

b) Process network extended with alternatives

Fig. 1. Structure representation of the suit jacket manufacturing problem

\section{Mathematical programming model}

Let us consider the following nonempty sets of $A, E$, and $V$,

$G(A, E, V) ; \quad A \cap E=0, \quad V \subseteq(A \times E) \cup(E \times A), \quad$ bipartitegraph, 
$A=\{i \in N\}$ activities,

$E=\{j \in N\}$ events.

In the current example the above sets have the following elements, see Fig. 1b:

$A=\{2,3,4,5,6,7,8,9,10,11,20,22,24,26,28,30,32,34,35$, close $\}$;

$E=\{1,12,13,14,15,16,17,18,19,21,23,25,27,29,31,33,36$, end $\}$

$V=\{(1,2),(1,3),(1,4),(1,5),(1,6),(1,7),(1,8),(1,9),(1,10),(1,11),(2,12),(3,13)$, $(4,14),(5,14),(6,15),(7,16),(8,17),(9,18),(10,18),(11,19),(19,20),(18,20)$, (20,21), (21,22), (17,22), (22,23), (23,24), (16,24), (24,25), (25,26), (15,26), (26,27), $(27,28),(14,28),(28,29),(29,30),(13,30),(30,31),(31,32),(12,32),(32,33),(33,34)$, $(33,35),(34,36),(35,36),(36$, close $)$, (close, end) $\}$.

Please note that the alternative operating units are denoted by nodes 4,10 , and 34 . Let us denote by $x_{i}$ the $i$-th operating unit in the process network depicted in Fig. $1 b$, where

$x_{i}=\left\{\begin{array}{ll}0, & \text { the } i-\text { th activity is not performed, } \\ 1, & \text { the } i-\text { th activity is performed. }\end{array}\right\}$

Other parameters of the mathematical programming model are the following: $t_{i}$ is the time from start-up to the $i$-th event occurs, as a result of the solution of the mathematical programming model; $T_{i}$ is the duration of the $i$-th activity as given in Table I; $C_{i}$ is the cost of the $i$-th activity as given in Table I; and $C$ is the planned upper budget for the total project.

\subsection{Solution of the original case without alternatives}

In the original case without alternatives, the following elements that refer to the alternatives are excluded from the above lists:

$$
\begin{aligned}
& A:=A \backslash\{4,10,34\} ; \text { and } \\
& V:=V \backslash\{(1,4),(4,14),(1,10),(10,18),(33,34),(34,36)\} .
\end{aligned}
$$

The mathematical programming model is

$$
\begin{aligned}
& x_{\text {close }}=1, \\
& t_{1}=0,
\end{aligned}
$$




$$
\begin{aligned}
& t_{j} \geq t_{k}+x_{i} T_{i}, \quad \forall j \in E \text { and } \forall i:(i, j) \in V \text { and } \forall k:(k, i) \in V, \\
& \qquad \begin{array}{c}
\sum x_{j} \geq x_{i} \\
\{j: \exists k, \text { where }(j, k) \text { and }(k, i) \in V\}
\end{array}, \quad \forall i \in A, \\
& t_{\text {end }} \rightarrow \text { min }
\end{aligned}
$$

Please note that Eq. (1) refers to the fact that the project has to be finished. Eq. (2) gives that the project starts at zero. Eq. (3) refers to the fact that the activities are completed at the earliest possible time, while Eq. (4) expresses the fact that when an activity is part of the solution then every predecessor activity has to be finished. Eq. (5) indicates that the aim is to minimize the overall duration of the project.

The solution of the above problem equals to the original case without alternatives, i.e. the original CPM problem. For this case the overall duration of the project is $7277 \mathrm{sec}$. The total working time of the project, when not only the shortest path is considered but the time of all operating units is: $10633 \mathrm{sec}$. The cost of the project for this case is: 7281 HUF.

\subsection{Solution of the case with alternatives and no cost constraints}

Let us consider the original sets $A$ and $V$; in other words, alternative operating units denoted by nodes 4,10 , and 34 are elements of $A$ and their connecting arcs are elements of $V$. The corresponding mathematical programming model consists of the same equations as above, i.e. Eq. (1) through Eq. (5), and there is an additional constraint Eq. (6). Please note that Eq. (6) indicates that in the project only one alternative can be considered, and Eq. (6) is a stronger constraint than Eq. (4), thus Eq. (4) can be eliminated or neglected

$$
\underset{\{i: i \in A \text { and }(i, j) \in V\}}{\sum x_{j}=1}, \quad \forall j \in E .
$$

For this case the overall duration of the project is: $6422 \mathrm{sec}$. Please note that $\mathrm{x}_{5}$ : original, $x_{10}$ : alternative and $x_{34}$ : alternative operating units are working in this case. The total working time of the project, when not only the shortest path is considered but the time of all operating units is: $9925 \mathrm{sec}$. The cost of the project for this case is: 7233 HUF.

\subsection{Solution of the case with alternatives and cost constraints}

Let us consider the original sets $A$ and $V$; in other words, alternative operating units denoted by nodes 4,10 and 34 are elements of $A$ and their connecting arcs are elements of $V$. The mathematical programming model is:

$$
x_{\text {close }}=1 \text {, }
$$




$$
\begin{aligned}
& t_{1}=0, \\
& t_{j} \geq t_{k}+x_{i} \cdot T_{i}, \quad \forall j \in E, \quad \forall i:(i, j) \in V \quad \text { and } \quad \forall k:(k, i) \in V, \\
& \sum_{\{i: i \in A \text { and }(i, j) \in V\}} x_{i}=1, \quad \forall j \in E, \\
& \sum_{\{i: i \in A\}} x_{i} \cdot C_{i} \leq C, \\
& t_{\text {end }} \rightarrow \min .
\end{aligned}
$$

Please note that in this mathematical programming model Eq. (7) through Eq. (12) is similar to the previously mentioned equations. The additional constraint, Eq. (11), indicates that the project cost should not exceed the given upper budget limit, which is set to be 7000 HUF for this case.

For this case the overall duration of the project is: $6422 \mathrm{sec}$. Everywhere the alternative operating units are working. The total working time of the project, where not only the shortest path is considered but the time of all operating units is: $10064 \mathrm{sec}$. The cost of the project for this case is: 6889 HUF. It is worth mentioning that the total working time is longer, yet the cost of the project is lower and the overall duration of the project (the critical path) is the same.

\section{Conclusion}

In the current paper a Hungarian clothing manufacturer's problem was presented; its corresponding CPM graph was depicted then transformed into a process network. Then, alternatives were added, exploiting the advantages of this structural representation; i.e. separate cases can commonly be handled within the resultant sole process network. Three different mathematical programming models were given together with solutions of the considered suit jacket problem.

\section{Acknowledgement}

The present scientific contribution is dedicated to the 650th anniversary of the foundation of the University of Pécs, Hungary.

\section{References}

[1] Chanas S., Zielinski P. Critical path analysis in the network with fuzzy activity times, Fuzzy Sets and Systems, Vol. 122, No. 2, 2001, pp. 195-204. 
[2] Guerriero F., Talarico L. A solution approach to find the critical path in a time constrained activity network, Comp. Oper. Res. Vol. 37, No. 9, 2010, pp. 1557-1569.

[3] Li Z., Liu Y., Yang G. A new probability model for insuring critical path problem with heuristic algorithm, Neurocomputing, Vol. 148, 2015, pp. 129-135.

[4] Szendröi E. A robust hybrid method for the multimode resource-constrained project scheduling problem, Pollack Periodica, Vol. 5, No. 3, 2010, pp. 175-184.

[5] Csébfalvi A., Láng B. An improved hybrid method for the resource-constrained project scheduling problem with discounted cash flows, Pollack Periodica, Vol. 7, No. 1, 2012, pp. 135-146.

[6] Danka S. A statistically correct methodology to compare metaheuristics in resource constrained project scheduling, Pollack Periodica, Vol. 8, No. 3, 2013, pp. 119-126.

[7] Liu Z., Xiao L., Tian J. An activity-list-based nested partitions algorithm for resourceconstrained project scheduling, International Journal of Production Research, Vol. 54, No. 16, 2015, pp. 4744-4758.

[8] Friedler F., Tarjan K., Huang Y. W., Fan L. T. Graph-theoretic approach to process synthesis: Axioms and theorems, Chem. Eng. Sci, Vol. 47, No. 8, 1992, pp. 1973-1988.

[9] Friedler, F., Tarjan K., Huang Y. W., Fan L. T. Combinatorial algorithms for process synthesis, Comp. Chem. Eng, Vol. 16, Supl. 1, 1992, pp. S313-S320.

[10] Tick J., Imreh Cs., Kovács Z. Business process modeling and the robust PNS problem, Acta Polytechnica, Vol. 10, No. 6, 2013, pp. 193-204.

[11] Kovacs Z., Ercsey Z., Friedler F., Fan L. T. Exact super-structure for the synthesis of separation-networks with multiple feed-streams and sharp separators, Comp. Chem. Eng. Vol. 23, No. Supplement 1, 1999, pp. S1007-S1010.

[12] Kovacs Z., Ercsey Z., Friedler F., Fan L. T. Separation-network synthesis: Global optimum through rigorous super-structure, Comp. Chem. Eng. Vol. 24, No. 8, 2000, pp. 1881-1900.

[13] Mandl M., Gwehenberger G., Narodoslawsky M., Krotscheck C., Birnstingl-Gottinger B. KOMEOS - Konzeption von Modellen multifunktionaler Energiezentren für die OstSteiermark, Bundesministerium für Verkehr, Innovation und Technologie, Austria, 2010.

[14] König É., Kalauz K., Bertók B. Synthesizing flexible process networks by two stage Pgraphs, Comp. Aided Chem. Eng, Vol. 33, 2014, pp. 1339-44.

[15] Imreh C., Kovács Z. On pollution minimization in the optimization models of process network synthesis, Chem. Eng. Trans, Vol. 7, No. 2, 2005, pp. 565-570.

[16] Kollmann R., Maier S., Shahzad K., Kretschmer F., Neugebauer G., Stoeglehner G., Ertl T., Narodoslawsky M., Waste water treatment plants as regional energy cells, evaluation of economic and ecologic potentials in Austria, Chemical Engineering Transactions, Vol. 39, 2014, pp. 607-612.

[17] Vincze N., Ercsey Zs., Kovács T., Tick J., Kovács Z. Process solution of extended CPM problems with alternatives, Acta Polytechnica Hungarica, Vol. 13, No. 3, 2016, pp. 101-117. 